\title{
Treatment of rheumatoid arthritis patients - a challenge
}

\author{
Luiza Maria Pana', Daniel Nicolae Costea', Razvan Adrian Ionescu ${ }^{1,2}$ \\ ${ }^{1}$ Colentina Clinical Hospital, Bucharest, Romania \\ 2 "Carol Davila" University of Medicine and Pharmacy, Bucharest, Romania
} \begin{abstract}
Rheumatoid arthritis (RA) is a chronic, systemic inflammatory autoimmune disease with unclear etiology that
involves the peripheral joints symmetrically, causing synovial hypertrophy, inflammation and, consequently, ero-
sions of the bone and cartilage. We hereby present the case of a 51-year-old female patient diagnosed with RA
in 2009 for which she received treatment with Methotrexate and TNF alpha treatment (Infliximab). Prior to the first
dose of Infliximab Quantiferon-TB Gold was performed with a negative result. In 2016 the patient presented with
ascites and pleural effusion with a positive repeated test for Quantiferon-TB Gold, the treatment with Infliximab
and Methotrexate was stopped and she was started on anti TB standard treatment regimen for 9 months with the
remission of the polyserositis. After the completion of the anti TB treatment we reinitiated Methotrexate therapy
given a high disease activity (DAS28-CRP of 6.59) which improved the patients complaints; however, 3 months
later, given an increase in the patients inflammatory markers we performed a computed tomography (CT) scan
that revealed peritoneal thickening, and several omental nodules, the histological examination confirming the
diagnosis of peritoneal TB. The patient was reinitiated on the anti-TB treatment.
Keywords: rheumatoid arthritis, anti-tumor necrosis alpha anti TNF alpha, Infliximab,
monoclonal antibodies, peritoneal tuberculosis
\end{abstract}
\section{INTRODUCTION}

Rheumatoid arthritis (RA) is a chronic, systemic inflammatory autoimmune disease with unclear etiology that involves the peripheral joints symmetrically, causing synovial hypertrophy, inflammation and, consequently, erosions of the bone and cartilage. Along its course, RA may also present with a series of extraarticular manifestations. Anti tumor necrosis alpha (TNF-alpha) biological agents are effective in the treatment of RA, however they increase the risk of infections and specifically Mycobacterium tuberculosis (TB) (1).

The development of anti-TNF alpha agents revolutionised the treatment paradigm in the field of inflammatory immune mediated rheumatic diseases including RA unresponsive or with a poor response to conventional disease-modifying antirheumatic drugs (DMARDs) like Methotrexate. Anti TNF-alpha treatment targets a central cytokine in the in- flammatory process relieving symptoms and influencing the course of the disease by reducing further structural disease progression and extraarticular manifestations. However, TNF-alpha also plays a key role in the defence against infectious agents, particularly intracellular organisms such as Mycobacterium tuberculosis. It has been stated that anti TNF-alpha monoclonal antibodies impose a greater risk of developing TB rather than TNF-alpha soluble receptor and also, a shorter time period between the first administration and the TB infection diagnosis $(2,3)$. The Romanian protocol for the treatment of rheumatoid arthritis patients with biologic agents requires the assessment of the risk of reactivating a latent TB infection. This assessment consists of a thorough patient history, clinical examination, chest X-ray, and Quantiferon TB Gold or a PPD test. All patients testing positive for interferon-gamma release assays undergo a pneumological consult and 
prophylactic treatment with isoniazid conducted by the pneumology specialist with the possibility of him issuing an approval for initiating biologic treatment after one month of prophylactic treatment. Only for patients with a negative initial screening test it is recommended to repeat the same initial screen test every year.

\section{CASE PRESENTATION}

A 51-year-old-female patient with a personal medical history consisting of seropositive rheumatoid arthritis eight years prior to the first admission for which she received initial treatment with the conventional DMARDSs (Methotrexate $15 \mathrm{mg}$ every week, Sulfasalasine $3 \mathrm{~g}$ per day) with a poor clinical and biologic response. The patient was screened for latent TB infection and viral hepatitis according to

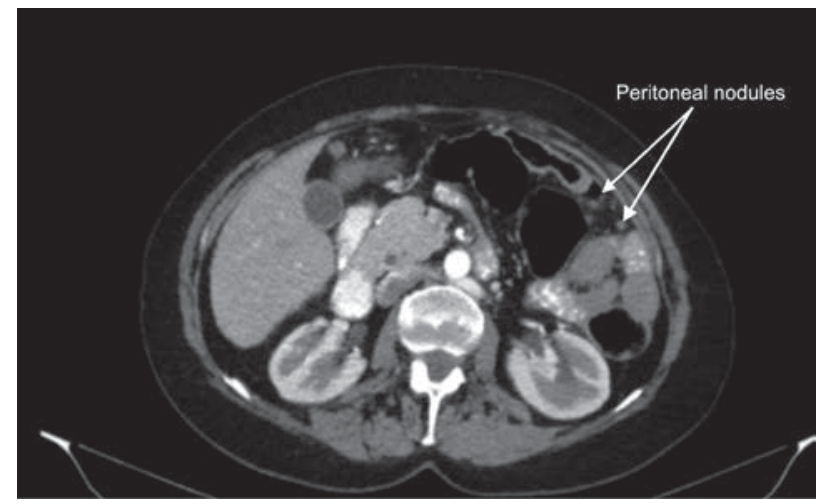

FIGURE 1. Peritoneal nodules on CT scan - transverse section

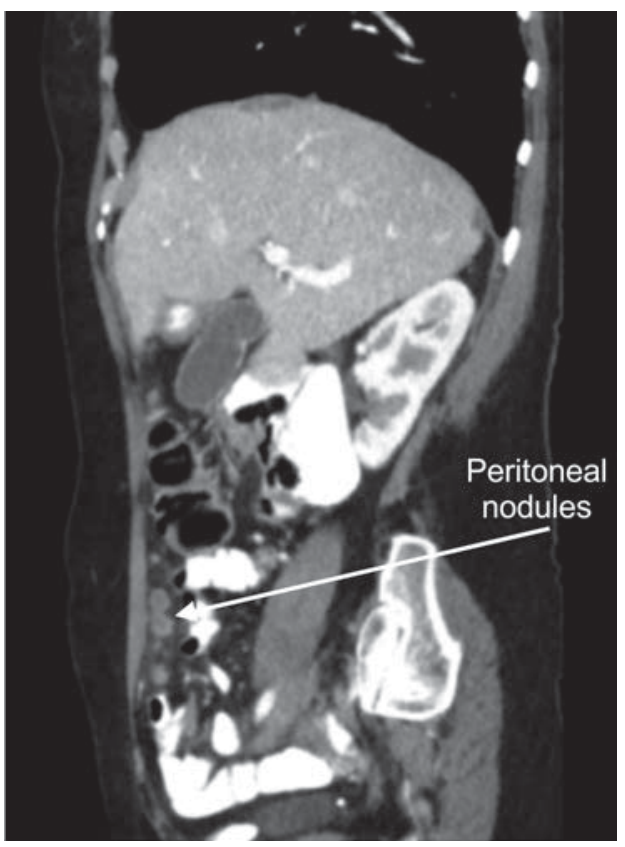

FIGURE 2. Peritoneal nodules on CT scan sagittal view
Romanian protocol for the treatment of rheumatoid arthritis patients with biologic agents by performing a chest X-ray that did not reveal abnormalities and Quantiferon-TB Gold assay that was negative. The patient was started on anti TNF-alpha therapy with Infliximab $3 \mathrm{mg} / \mathrm{kg}$ according to the RA treatment protocol, with a positive response, decrease of DAS 28-CRP from 5.2 to 2.5 after 3 months of treatment. Seven years later she was diagnosed with deep venous thrombosis (DVT) of the femoral and popliteal veins. The patient tested positive for lupus anticoagulant factor at the diagnosis of the DVT and 12 weeks after the first test, therefore, she was diagnosed with antiphospholipid syndrome and she was started on acenocumarol treatment. Two months later the patient presented with exudative lymphocytic ascites and pleural effusion with positive adenosine deaminase, negative cultures and PCR for M. tuberculosis. However, the patient converted positive for the Quantiferon-TB Gold assay. Both chest X-Ray and thoracic, abdominal and pelvic CT scan revealed no lesions other than the pleural and peritoneal effusion. Given this clinical picture we concluded that in spite of lacking direct evidence of the M. tuberculosis infection, a conversion of the interferon-gamma release assay in a patient with polyserositis receiving Infliximab treatment is highly indicative of extrapulmonary TB. We stopped the treatment with both conventional synthetic and biologic DMARDs and she was started on anti TB standard therapy with isoniazid $300 \mathrm{mg} /$ day, ethambutol $800 \mathrm{mg} /$ day, pyrazinamide $1,500 \mathrm{mg} /$ day and rifampicin $600 \mathrm{mg} /$ day for 9 months in the Pneumology Department. Following the completion of the treatment plan, given the approval from the Pneumologist and a high disease activity (DAS28-CRP of 7.2) we reinitiated the treatment with Methotrexate. After 3 months of csDMARD treatment, in spite of a clear improving in the patients complaints, and in the clinical and ultrasound articular findings, given very high inflammatory markers (ESR $97 \mathrm{~mm} / \mathrm{h}, \mathrm{CRP}=120 \mathrm{mg} / \mathrm{l}$ with normal values of $0-20 \mathrm{mmm} / \mathrm{h}$ for ESR and $0-5$ $\mathrm{mg} / \mathrm{l}$ for the CRP), we performed a thoracic, abdominal and pelvic computed tomography (CT) scan that revealed peritoneal thickening, and several omental nodules. Laparoscopy and biopsy of the nodules revealed several granulomas with extended caseous necrosis bordered by several epitheliod histiocytes and Langhans giant cells and numerous lymphocites confirming the diagnosis of peritoneal TB. The patient was refered back to the Pneumology 


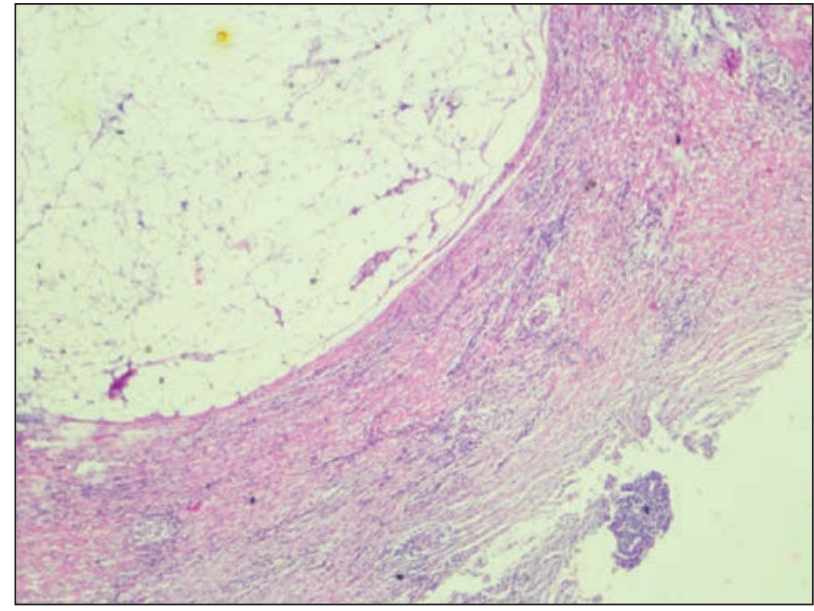

FIGURE 3. Fatty tissue and inflammatory cells $4 x$

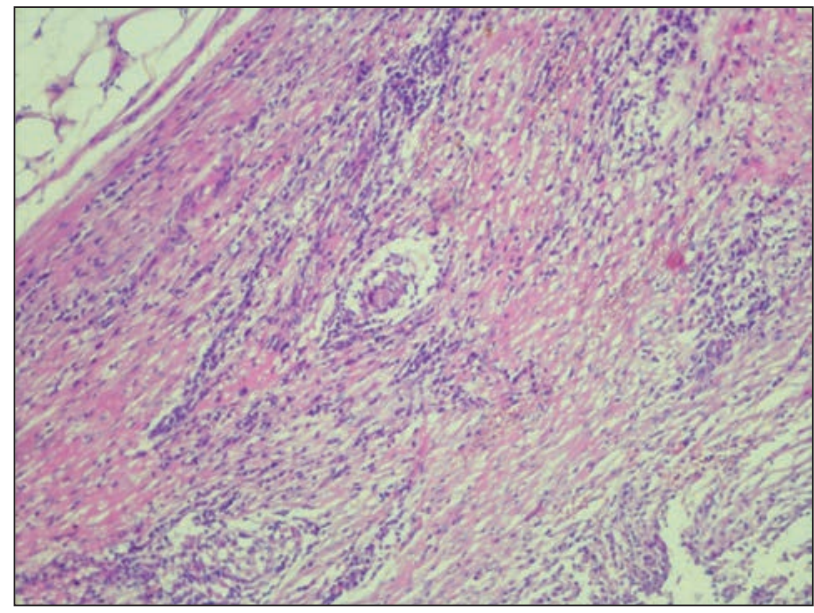

FIGURE 4. Langhans cell 10x

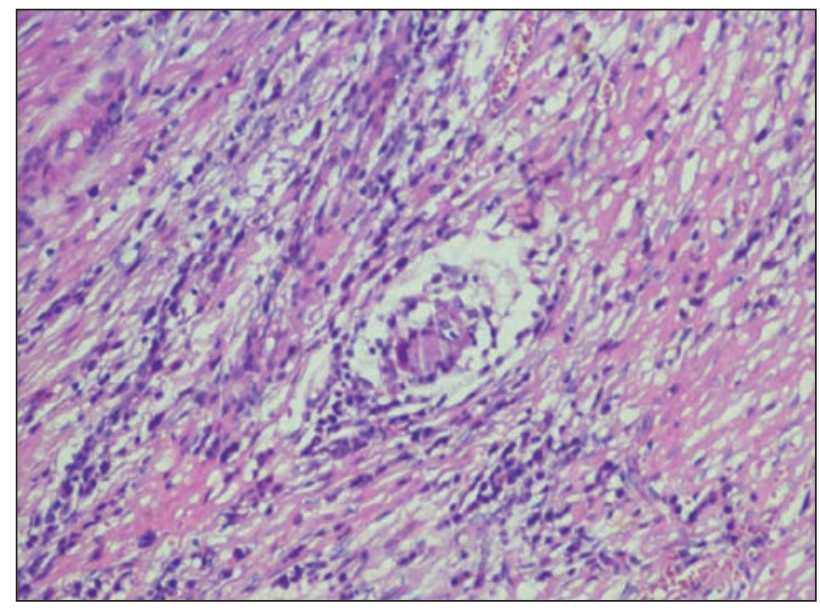

FIGURE 5. Langhans Cell 20x

Department where she was reinitiated on anti-TB therapy according to the TB treatment protocol.

\section{DISCUSSION}

TNF alpha is cytokine with a key-role in the pathogenic inflammatory cascade of several immune mediated inflammatory disease such as rheumatoid

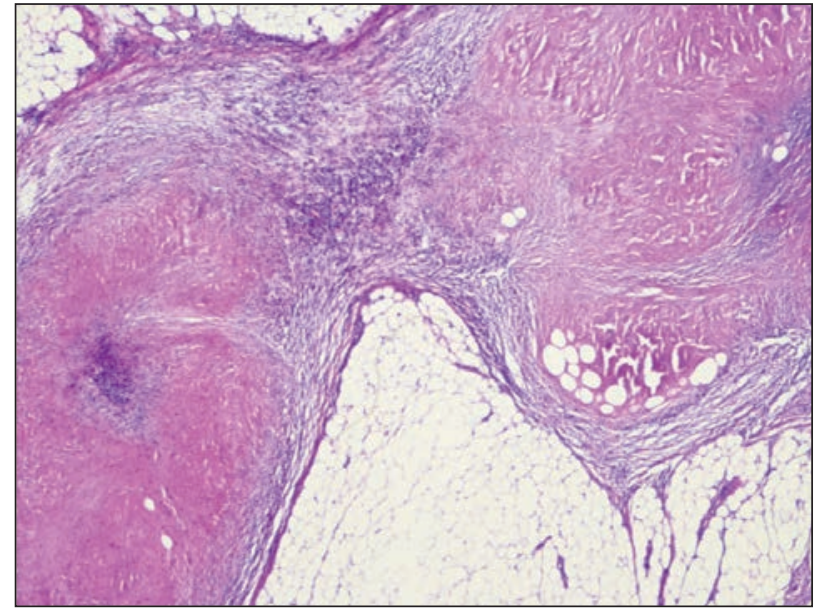

FIGURE 6. Confluent areas of necrosis

arthritis. Blocking the TNF-alpha related pathways represented a paradigm shift in the treatment and progression of the disease. However, given the involvement of TNF in several physiological mechanisms such as antimicrobial response, particularly against intracellular microorganisms like $M$. tuberculosis (4). Several data indicate that TNF plays an important role in the formation of the granuloma, a key element in limiting $M$. tuberculosis infection progression, thus, blocking the TNF pathways may lead to extensive and more rapidly progressing forms of disease in extrapulmonary sites $(4,5)$.

Blocking TNF alpha may be obtained by using to types of molecules: monoclonal antibodies either chimeric (infliximab) or human monoclonal antibodies (adalimumab, golimumab etc) or a fusion protein between human TNF receptor 2 and the Fc fragment of human IgG1 (etanercept). Monoclonal antibodies are 5 to 10 times more likely to reactivate a latent $M$. tuberculosis infection compared to the soluble receptor (6).

M. tuberculosis infection is a major public health problem in Romania. In spite of the constant decrease in new cases after 2002 due to more effective national prophylaxis, diagnosis and treatment program, the incidence of 68:1,000 is still the highest in the European Uninon, remaining a major threat regarding mortality and morbidity, particularly in the setting of increasing population migration (7).

Given the increased risk for M. tuberculosis in Romania, the Romanian protocol for the treatment of Rheumatoid arthritis patients with biologic agents requires the assessment of the risk of reactivating a latent TB infection. This assessment consists of a thorough patient history, clinical examination, chest X-Ray, and Quantiferon TB Gold or a PPD test. All 
patients testing positive for interferon-gamma release assays undergo a pneumological consult and prophylactic treatment with isoniazid conducted by the pneumology specialist with the possibility of him issuing an approval for initiating biologic treatment after one month of prophylactic treatment. Only for patients with a negative initial screening test it is recommended to repeat the same initial screen test every year together with a chest X-ray. However, across other E.U. countries with a low incidence of $M$. tuberculosis infection and consequently with low immunisation rates, there is no consensus regarding the optimal method for screening and particularly in the monitoring the potential development of TB during biologic treatment. We believe this might become an important public health issue, in the light of the increasing migration from countries endemic for TB infection and, therefore a coherent unified immunisation, screening and monitoring strategy should be taken into consideration. Of course, there still remains the problem of what drug should this patient receive as DMARD, in order to control her RA and to preserve a good functioning and a good quality of life for the patient. After complete resolution of the specific infectious process and only upon having the pulmonologist's approval, we will appreciate if the activity of the rheumatological disease is such that we might try to control it only with conventional synthetic DMARDs (in which case, we will probably choose Sulfasalazine) or it will be necessary to use a biological DMARD. If so, as stated above, since fusion soluble receptor molecules biologics are less probable in activating a M. tuberculosis infection, we will prefer this kind of therapeutic agent over other types. Such a patient must remain in close contact with and active watching by the rheumatologist, but by the pulmonologist, as well, since these are intricated pathologies occurring in the same patient. This is a typical case of much needed collaboration between different specialities, under the coordination of the rheumatologist, as stated in the EULAR guidelines as well (8), in order to provide the patient with the best possible care and outcomes.

\section{CONCLUSIONS}

TNF alpha antagonists are effective therapeutic options for RA, however, particularly monoclonal antibodies increase the risk of developing $M$. tuberculosis infection including extrapulmonary extensive forms which are harder to diagnose and treat.

Prophylaxis and screening before initiating biologic treatment and periodic monitoring for the potential development of TB should be major concerns even in countries with low incidences for $M$. tuberculosis.

Conflict of interest: none declared Financial support: none declared

\section{REFERENCES}

1. Xie X, Li F, Chen JW, Wang J. Risk of tuberculosis infection in anti-TNF-a biological therapy: from bench to bedside. J Microbiol Immunol Infect. 2014;47(4):268-74.

2. Tubach F, Salmon D, Ravaud $P$, Allanore $Y$, Goupille $P$, Breban $M$, et al. Risk of tuberculosis is higher with anti-tumor necrosis factor monoclonal antibody therapy than with soluble tumor necrosis factor receptor therapy: the three-year prospective French Research Axed on Tolerance of Biotherapies registry. Arthritis Rheum. 2009;60:1884e94.

3. Brassard P, Kezouh A, Suissa S. Antirheumatic drugs and the risk of tuberculosis. Clin Infect Dis. 2006;43:717e22.

4. Kindler V, Sappino AP, Grau GE, Piguet PF, Vassalli P. The inducing role of tumor necrosis factor in the development of bactericidal granulomas during BCG infection. Cell. 1989;56(5):731-40.
5. Solovic I, Sester M, Gomez-Reino JJ, Rieder HL, Ehlers S, Milburn HJ, Kampmann B et al. The risk of tuberculosis related to tumour necrosis factor antagonist therapies: a TBNET consensus statement. Eur Respir J. 2010;36(5):1185-206.

6. Mezouar S, Diarra I, Roudier J, Desnues B, Mege JL. Tumor Necrosis Factor-Alpha Antagonist Interferes With the Formation of Granulomatous Multinucleated Giant Cells: New Insights Into Mycobacterium tuberculosis Infection. Front Immunol. 2019;10:1947.

7. https://www.ecdc.europa.eu/sites/portal/files/documents/

8. Smolen JS, Landewe RBM, Biljsma JWJ, et al. EULAR recommendations for the management of rheumatoid arthritis with synthetic and biological disease-modifying antirheumatic drugs: 2019 update. Ann Rheum Dis. 2020;79:685-699. tuberculosis-surveillance-monitoring-Europe-2019-20_Mar_2019 